\title{
CARACTERÍSTICAS DA CONVECÇÃO ASSOCIADA À VARIABILIDADE INTRASSAZONAL: COMPARAÇÃO ENTRE RESULTADOS DO MCGA CPTEC/COLA E OBSERVAÇÃO
}

\author{
GLAUCIA MEIRA CARNEIRO E IRACEMA FONSECA ALBUQUERQUE CAVALCANTI
}

\author{
Instituto Nacional de Pesquisas Espaciais (INPE), Cachoeira Paulista, SP, Brasil \\ glaucia.meira@cptec.inpe.br, iracema.cavalcanti@cptec.inpe.br
}

Recebido Dezembro 2007 - Aceito - Abril 2007

\begin{abstract}
RESUMO
O desenvolvimento de técnicas de monitoramento da Variabilidade Intrassazonal (VI) sobre a América do Sul (AS), utilizando resultados de previsão sazonal com modelos numéricos, requer um estudo inicial do comportamento dos modelos na representação da VI em simulações climáticas. Neste estudo foi feita uma comparação entre os dados observados e os obtidos de uma simulação climática de longo prazo com o Modelo de Circulação Geral Atmosférico (MCGA) CPTEC/COLA, para o verão no período de 1981 a 2001, ambos filtrados na banda de 30 a 90 dias. Características semelhantes foram identificadas nos dois conjuntos de dados nos campos climatológicos e de variância de Radiação de Onda Longa Emergente (ROL), embora com intensidades diferentes. O MCGA representou razoavelmente bem algumas características da VI nos dados de ROL, as quais representam a variabilidade na convecção. Na região tropical, o padrão típico observado da Oscilação de Madden e Julian (OMJ) não é representado adequadamente pelo modelo, embora este apresente um padrão alternado de anomalias na região da Indonésia e Pacífico. As configurações de VI quando a análise é feita apenas sobre a AS, tais como o dipolo de convecção associado à Zona de Convergência do Atlântico Sul, são bem simuladas.

Palavras Chave: Variabilidade Intrassazonal, Radiação de Onda Longa Emergente, Simulação Climática, MCGA.

ABSTRACT: CONVECTION FEATURES ASSOCIATED WITH INTRASEASONAL VARIABILITY: A COMPARISON BETWEEN CPTEC/COLA AGCM RESULTS AND OBSERVATION.

Monitoring Intraseasonal Variability (IV) and its influence over South America requires an appropriate investigation of the correct representation of IV in climate simulations. Preliminary analysis of the CPTEC/COLA AGCM simulations indicated that this model captures the main modes of IV that affect South America. In the present study a comparison between observed and simulated 30-90 days filtered Outgoing Longwave Radiation (OLR) anomalies was performed for the 1981-2001 summer season. Similar climatological and variance fields characteristics were identified in both data sets, although with different intensities. Some OLR IV characteristics, representing convection variability were well represented by the CPTEC/COLA AGCM. The typical MJO observed pattern in the tropical region is not properly represented by the model, although an alternate OLR anomaly pattern is seen over Indonesia and Pacific Ocean. However the IV patterns, such as the convection dipole associated with the South Atlantic Convergence Zone, are well simulated when the analysis is performed only over South America.
\end{abstract}

Keywords: Intraseasonal Variability, South America convection, Climate Simulation, AGCM. 


\section{INTRODUÇÃO}

A Variabilidade Intrassazonal (VI) tem um papel importante nos regimes de precipitação sobre a América do Sul (AS), em especial sobre o Brasil. No período de verão, a Zona de Convergência do Atlântico Sul (ZCAS) sofre a ação da VI, ocasionando períodos de excesso ou de falta de precipitação nas Regiões Sudeste e Centro-Oeste do Brasil (Nogués-Paegle e Mo, 1997; Muza et al., 2009; Liebmann et al., 2004). A Região Nordeste também sofre influência da VI através da ação da Oscilação de Madden e Julian (OMJ), (Madden e Julian, 1994), a qual pode afetar o regime de chuvas na estação chuvosa dessa região (Kousky e Kayano, 1994).

Vários estudos documentam a influência de oscilações intrassazonais na convecção da AS, como Casarin e Kousky (1986), Kousky e Cavalcanti (1988), Kayano e Kousky (1992), Kousky e Kayano (1993 e 1994), Liebmann et al. (1999), Paegle et al. (2000), Carvalho et al. (2004), Cunningham e Cavalcanti (2006), Souza e Ambrizzi (2006), entre outros. Casarin e Kousky (1986) sugeriram uma relação entre o dipolo de oscilação de precipitação Sul-Sudeste e a OMJ. Kayano e Kousky (1992) e Kousky e Kayano (1994) identificaram um padrão de anomalias da Radiação de Onda Longa Emergente (ROL) sobre a AS, possivelmente associado com a oscilação 30-60 dias. Kousky e Kayano (1993) mostraram que as variações na convecção tropical estão relacionadas com anomalias na circulação de escala global. Liebmann et al. (1999) calcularam o espectro de ROL na região da ZCAS, e encontraram picos espectrais na banda subssazonal ( 2 a 30 dias) além da intrassazonal ( 30 a 90 dias). Eles analisaram a banda subssazonal e mostraram que o trem de ondas, que passa na região da ZCAS, segue aproximadamente a rota dos "grandes círculos", da região próxima à Nova Zelândia até as altas latitudes do Pacífico Sul e daí em direção ao equador na AS. Paegle et al. (2000) relacionaram o dipolo de convecção sobre a ZCAS com a OMJ e com um trem de ondas do Pacífico tropical em direção à AS, e se curvando para o nordeste sobre o continente. $\mathrm{O}$ estudo realizado por Carvalho et al. (2004) indica que a OMJ modula intensamente eventos de ZCAS com persistência maior que três dias. No estudo de Cunningham e Cavalcanti (2006) foram destacados dois mecanismos que influenciam a convecção na região da ZCAS na escala intrassazonal. $\mathrm{O}$ primeiro refere-se à propagação da OMJ para leste equatorialmente, da Indonésia para a região tropical da AS, quando a ZCAS encontra-se mais ao norte da posição climatológica. O segundo é a teleconexão representada pelo padrão "Pacific South America" (PSA) que influencia a convecção na ZCAS, quando a mesma encontra-se na posição climatológica ou mais ao sul da sua posição climatológica.

Os resultados de modelos numéricos de circulação geral da atmosfera apresentam uma previsibilidade alta na Região
Nordeste do Brasil (Marengo et al. 2003), a qual está sob a influência direta da forçante de Temperatura da Superfície do Mar (TSM). Entretanto, na Região Sudeste, esses modelos mostram baixa previsibilidade sazonal. Uma das razões sugeridas é que em um modelo atmosférico as TSM são as observadas e prescritas como condições de contorno, e assim a ZCAS sofreria a influência das TSM observadas mensalmente. Porém, como discutido em Chaves e Nobre (2004), a ZCAS tem uma influência sobre as TSM do Atlântico, em análises observacionais, através da redução da radiação solar, que resfriaria as águas superficiais nessa região. Como em um modelo atmosférico não há fluxos de calor entre o oceano e a atmosfera, esse aspecto observacional não é simulado. Algumas das características dos principais modos de VI, que afetam a AS e o Brasil, são bem representadas pelo MCGA CPTEC/ COLA, como visto em estudos preliminares com resultados de uma simulação climática de 10 anos (Cavalcanti e Castro, 2004). Uma dessas configurações é o padrão dipolo norte-sul de ROL, que indica variabilidade na intensidade da ZCAS. Como as anomalias de precipitação na região da ZCAS não são bem previstas na escala sazonal, é necessário extrair dos resultados, a componente intrassazonal, a qual tem influência nas anomalias. Na Região Nordeste a previsibilidade é maior do que nas outras regiões da AS, mas como é também afetada pela VI, esta região poderia se beneficiar de uma previsão que contivesse essa escala.

O objetivo deste artigo é fazer uma comparação da VI de ROL entre os resultados do MCGA CPTEC/COLA e a observação, analisando em detalhes algumas características sobre a AS para verificar a habilidade do modelo na representação dessa variabilidade. Essas informações serão úteis para aplicação de técnicas de monitoramento da influência da VI sobre diversas áreas do continente em resultados de previsão sazonal.

\section{DADOS E METODOLOGIA}

Foram utilizados dados diários observados de ROL da NOAA (Liebmann e Smith 1996) e dados diários de uma simulação climática com o MCGA CPTEC/COLA. Essa simulação consta de uma integração de 50 anos com o MCGA CPTEC/COLA na resolução de T62 L28 (aproximadamente $200 \mathrm{~km}$ entre os pontos de grade horizontais e 28 níveis na coordenada vertical). As TSMs mensais observadas foram utilizadas como condições de contorno e as condições iniciais utilizadas foram análises do NCEP. O período de estudo foi de 1981 a 2001, para a estação de verão (DJF). A variabilidade sazonal e características climatológicas simuladas pelo modelo, além dos detalhes do MCGA foram apresentadas em Cavalcanti et al. (2002). A variável ROL foi escolhida, porque nos permite 
inferir o comportamento da convecção sobre a AS, também usada em Kousky (1988), Liebmann et al. (1999), Carvalho et al. (2004), Cunningham e Cavalcanti (2006) e outros.

Inicialmente foram calculadas anomalias diárias dos dados observados e do modelo a partir da climatologia de 1981 a 2001. Após o cálculo das anomalias diárias de ROL, efetuou-se a filtragem dos dados de anomalias para reter a VI entre o período de 30 a 90 dias, com o objetivo de eliminar a variabilidade menor que 30 dias (escala mensal) e maior que 90 dias (escala sazonal). Essa banda, usada em Cavalcanti e Castro (2004) para analisar a VI em resultados do MCGA CPTEC/COLA, representou características observadas sobre a AS e oceanos adjacentes. O filtro utilizado nos resultados da simulação foi o Lanczos (Duchon, 1979). Embora a funçãoresposta do filtro Lanczos elimine parte da energia nos limites do filtro, este tem uma importante característica de reduzir as amplitudes das oscilações de Gibbs da série filtrada, e tem sido muito utilizado para filtragem de dados. Posteriormente foram analisadas as variâncias dos dois conjuntos de dados (observado e simulado) no Hemisfério Sul, como também a climatologia dos 21 anos de dados de ROL. A variância foi calculada por :

$$
\sigma=\sqrt{\frac{\sum_{i=1}^{n}\left(x_{i}-\bar{X}\right)^{2}}{n}}
$$

onde $\mathrm{x}_{\mathrm{i}}$ é o valor de ROL para cada dia, $\bar{X}$ é a média de 21 verões e $n=21$.

Para destacar as regiões em que a convecção (ROL) é afetada pela VI e identificar os padrões de variabilidade da convecção (ROL), foi aplicada a técnica de Funções Ortogonais Empíricas (EOF; Hannachi et al. 2007) em três regiões, uma que compreende todo o Hemisfério Sul $\left(10^{0} \mathrm{~N}-40^{0} \mathrm{~S} ; 0^{0}-360^{0} \mathrm{~W}\right)$, outra da região tropical $\left(10^{0} \mathrm{~N}-10^{0} \mathrm{~S} ; 60^{0} \mathrm{~W}-30^{0} \mathrm{~W}\right)$ e uma outra apenas da $\mathrm{AS}\left(10^{0} \mathrm{~N}-40^{\circ} \mathrm{S} ; 90^{0} \mathrm{~W}-20^{\circ} \mathrm{W}\right)$. Configurações da VI de convecção sobre a AS, associadas à intensa convecção em várias regiões do continente (Figura1), foram obtidas de compostos de casos com intensa convecção nessas regiões. Os casos foram selecionados considerando os dias com anomalias negativas de ROL acima do desvio padrão calculado para cada área da Figura 1, nos resultados do modelo e nas observações (Tabela I).

\section{ANÁLISES DE ROL NOS RESULTADOS DO MODELO E COMPARAÇÃO COM OBSERVAÇÃO}

\subsection{Climatologia Sazonal e Variância Intrassazonal no Hemisfério Sul}

No campo climatológico observado de ROL, no período do verão de 1981 a 2001 (Figura 2), considerando que os valores de ROL inferiores a $240 \mathrm{~W} / \mathrm{m}^{2}$ representam nebulosidade convectiva nos trópicos (Kousky, 1988), destacam-se três grandes áreas com grande atividade convectiva: uma sobre a

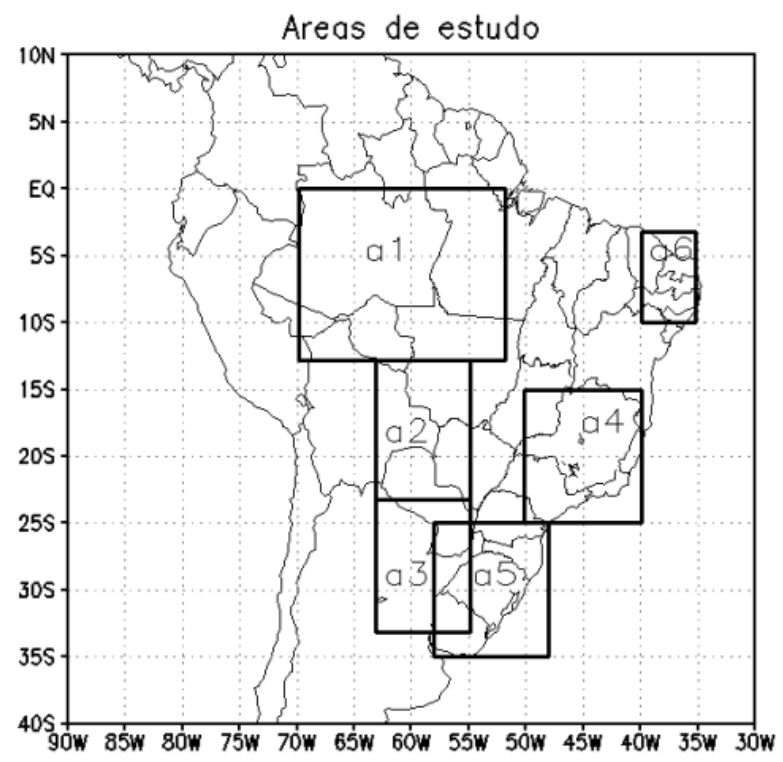

Figura 1 - Figura esquemática para identificar as áreas de estudo.

Tabela 1 - Valores do Desvio Padrão e o número de casos anômalos para cada área de estudo

\begin{tabular}{|c|c|c|c|c|}
\hline $\begin{array}{l}\text { Anomalia de ROL } \\
\text { Filtrs de Lanczos } \\
30-90\end{array}$ & $\begin{array}{c}\text { OBS } \\
\text { Desvio } \\
\text { Parlito }\end{array}$ & $\begin{array}{l}\text { MGGA } \\
\text { Desvio } \\
\text { Parlrío }\end{array}$ & $\begin{array}{c}\text { OBS } \\
\text { compostas } \\
\text { Qunntidade } \\
\text { de casas }\end{array}$ & $\begin{array}{c}\text { MOGA } \\
\text { compostos } \\
\text { Qugntidnde } \\
\text { de crags }\end{array}$ \\
\hline 11 & 9 & 7 & $10 s$ & 64 \\
\hline $\mathrm{az}$ & 11 & 11 & 159 & 161 \\
\hline D3 & 11 & 12 & 168 & 151 \\
\hline 104 & 17 & 14 & 194 & 115 \\
\hline 5 & 10 & 16 & 167 & 146 \\
\hline 106 & 12 & 9 & 224 & 237 \\
\hline
\end{tabular}


África, outra sobre a Indonésia e outra área sobre a AS. Uma outra observação é que sobre a AS, a convecção mais intensa é observada sobre o oeste da Amazônia e se estende para sudeste, refletindo também a atividade convectiva na região da ZCAS.

Comparando os resultados do MCGA com o observado, nota-se que o MCGA subestimou a convecção, principalmente, na região da Indonésia e sobre a parte oeste da AS. Um déficit de precipitação na Amazônia também foi encontrado na simulação climática de 10 anos com o mesmo modelo, apresentada em Cavalcanti et al. (2002). Sobre o Pacífico, a presença da Zona de Convergência do Pacífico Sul (ZCPS) é identificada nos resultados do modelo, porém com um deslocamento para leste em comparação com as observações, assim como a convecção sobre a África, que também se desloca para leste no modelo. Entretanto, a Zona de Convergência Intertropical (ZCIT) do Pacífico e do Atlântico apresentam uma superestimativa na convecção, embora a ZCIT do Atlântico seja simulada na posição observada.

A variância observada de ROL na banda intrassazonal apresenta valores máximos sobre a Indonésia e oceanos adjacentes, e na região da ZCAS (Figura 3). A variância obtida nos resultados da simulação é bem menor que a observada, embora as regiões de máximos valores sejam identificadas pelo modelo.

\subsection{Padrões de Variabilidade Intrassazonal no Hemisfério Sul, sobre a região tropical e sobre a América do Sul}

Na tabela II são apresentadas as porcentagens das variâncias explicadas para os 3 primeiros modos de variabilidade observada e simulada de anomalias de ROL na banda de 30 a 90

Tabela 1 - Valores do Desvio Padrão e o número de casos anômalos para cada área de estudo

\begin{tabular}{|c|c|c|c|c|}
\hline & \multicolumn{2}{|c|}{ Hemiglério Sul } & \multicolumn{2}{|c|}{ América do Sul } \\
\hline & OBS & MODELO & OBS & MODELO \\
\hline $\mathbf{1}^{\top}$ & $\mathbf{1 2 , 1}$ & 47 & $\overline{19,3}$ & 15,3 \\
\hline $\mathbf{2}$ & 11,2 & 4,2 & 12,11 & 9 \\
\hline $\mathbf{3}$ & 5,6 & 3,1 & 7,2 & 6,7 \\
\hline
\end{tabular}
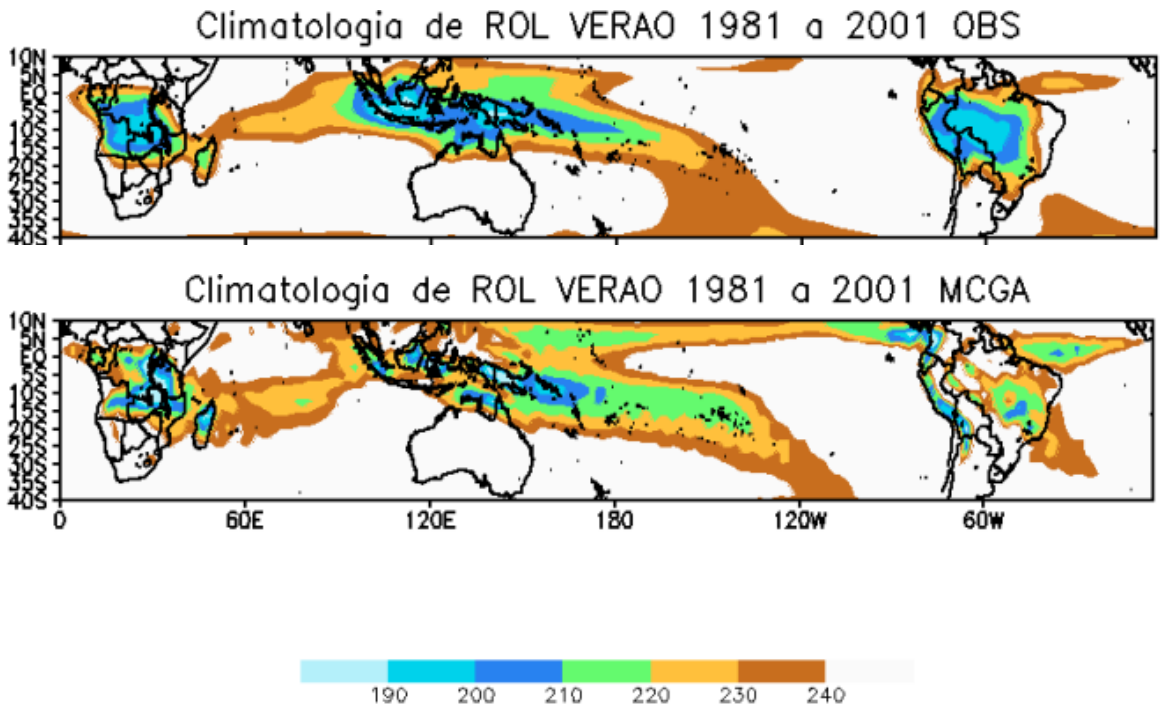

Figura 2 - Climatologia de ROL para o verão de 1981 a 2001. (a) OBS e (b) MCGA. 
dias. A variância é bem reduzida para o terceiro modo. Assim são apresentadas e discutidas apenas as configurações dos dois primeiros. Na análise observacional, o primeiro modo de VI representa bem o padrão típico da OMJ na região equatorial da Indonésia/Índico/Pacífico Oeste, além da variabilidade na região da ZCAS, ZCPS e ZCI (Figura 4a), enquanto o segundo representa a evolução da OMJ na região Índico/Indonésia / Pacífico Oeste e influências sobre a Região Nordeste do Brasil e sobre a África (Figura 4b). Essas configurações são semelhantes às obtidas em Cunningham e Cavalcanti (2006).
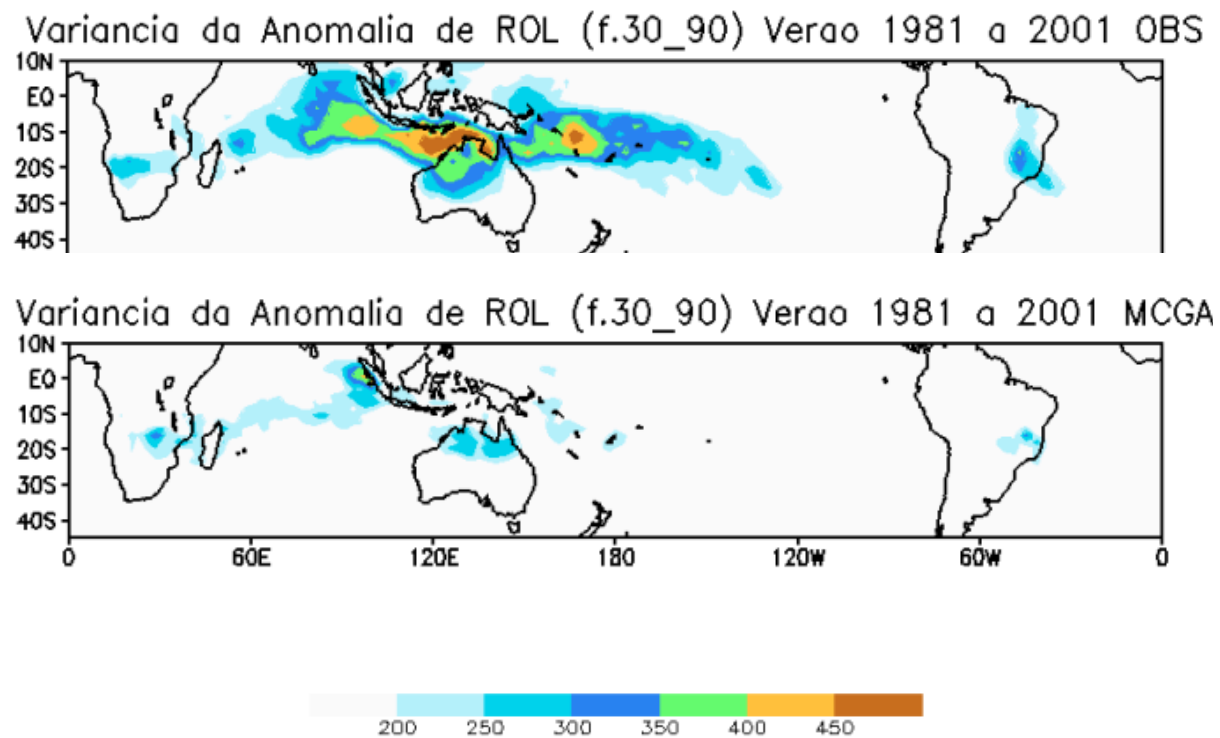

Figura 3 - Variância de ROL para os dados filtrados na banda intrasazonal, nos verões de 1981 a 2001. (a) OBS e (b) MCGA.
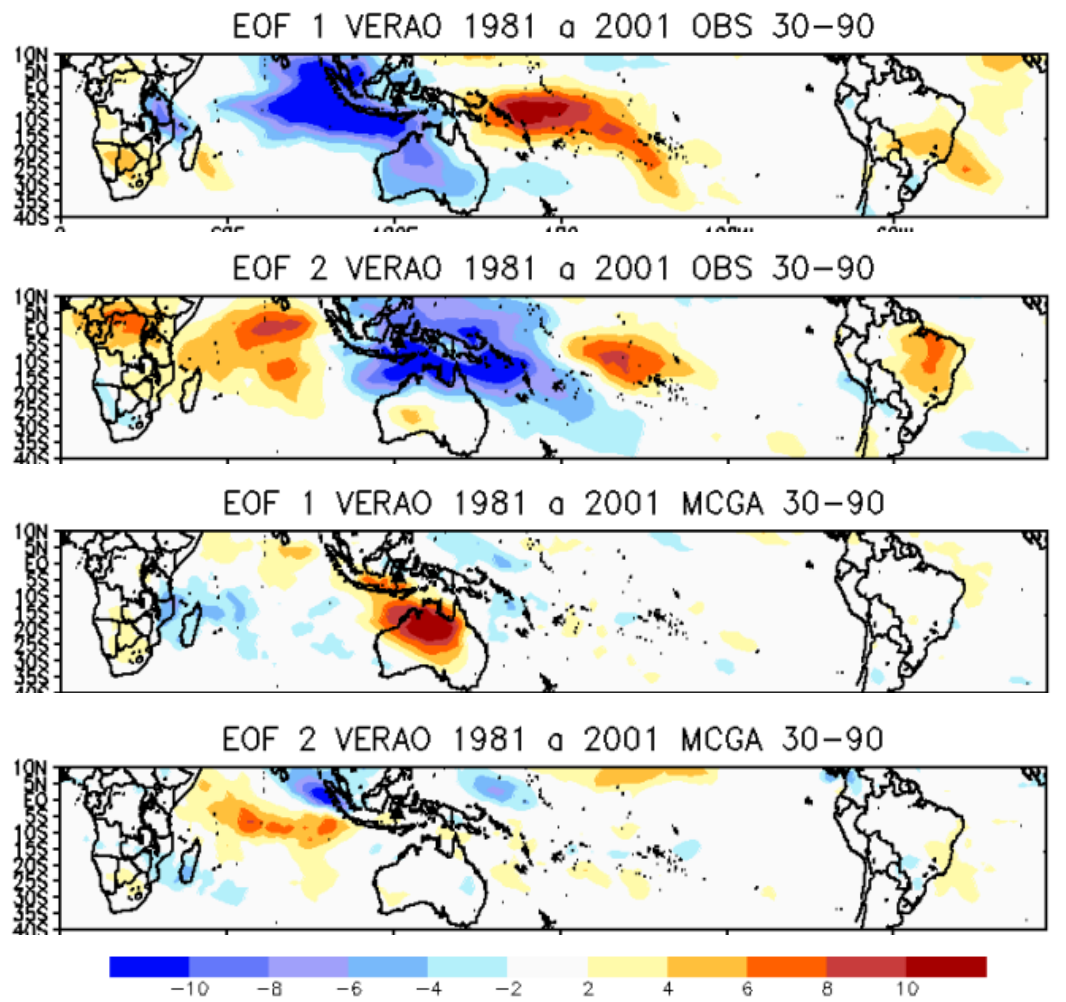

Figura 4 - EOF aplicado aos dados de anomalia de ROL na banda intrasazonal (30-90), para o verão de 1981 a 2001. (a) EOF1-OBS; (b) EOF2OBS; (c) EOF1-MCGA; (d) EOF2-MCGA. 


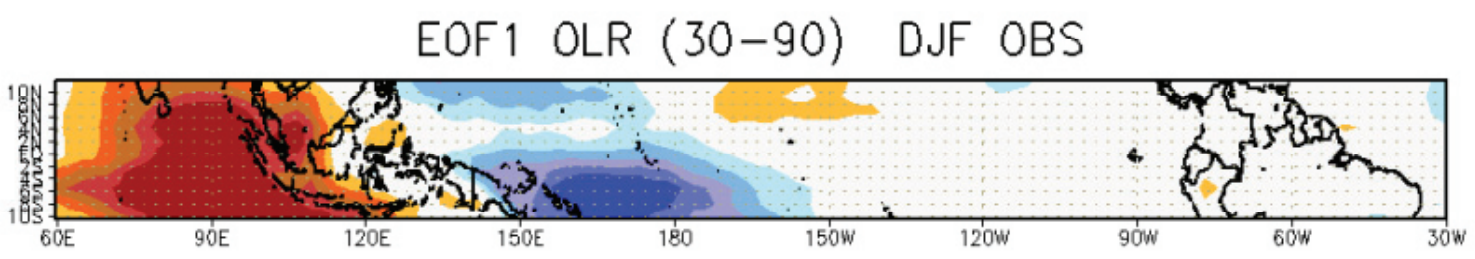

(a)

EOF2 OLR (30-90) DJJ OBS

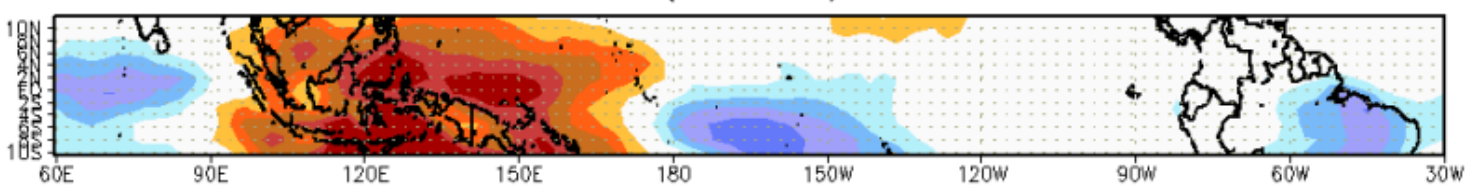

(b)

EOF1 OLR (30-90) DJF MCGA

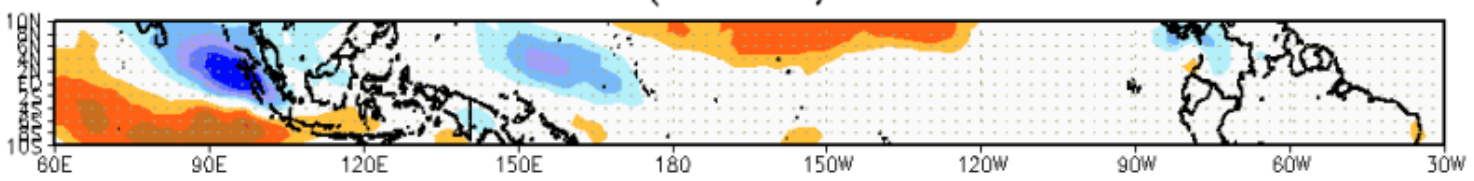

(c)

EOF2 OLR (30-90) DJF MCGA

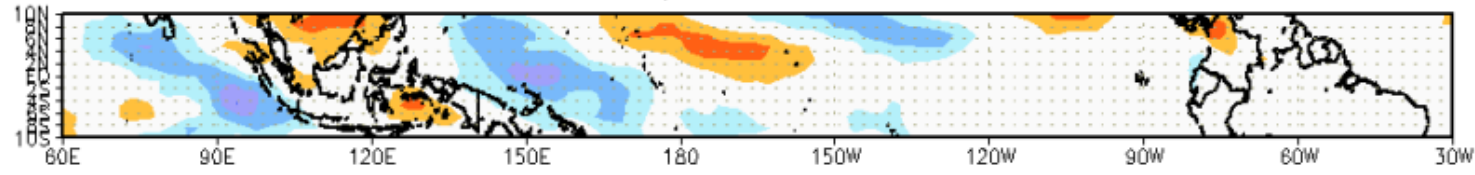

(d)

Figura 5 - EOF aplicado aos dados de anomalia de ROL na banda intrasazonal (30-90), para o verão de 1981 a 2001 na região tropical (a) EOF1OBS; (b) EOF2-OBS; (c) EOF1-MCGA; (d) EOF2-MCGA.
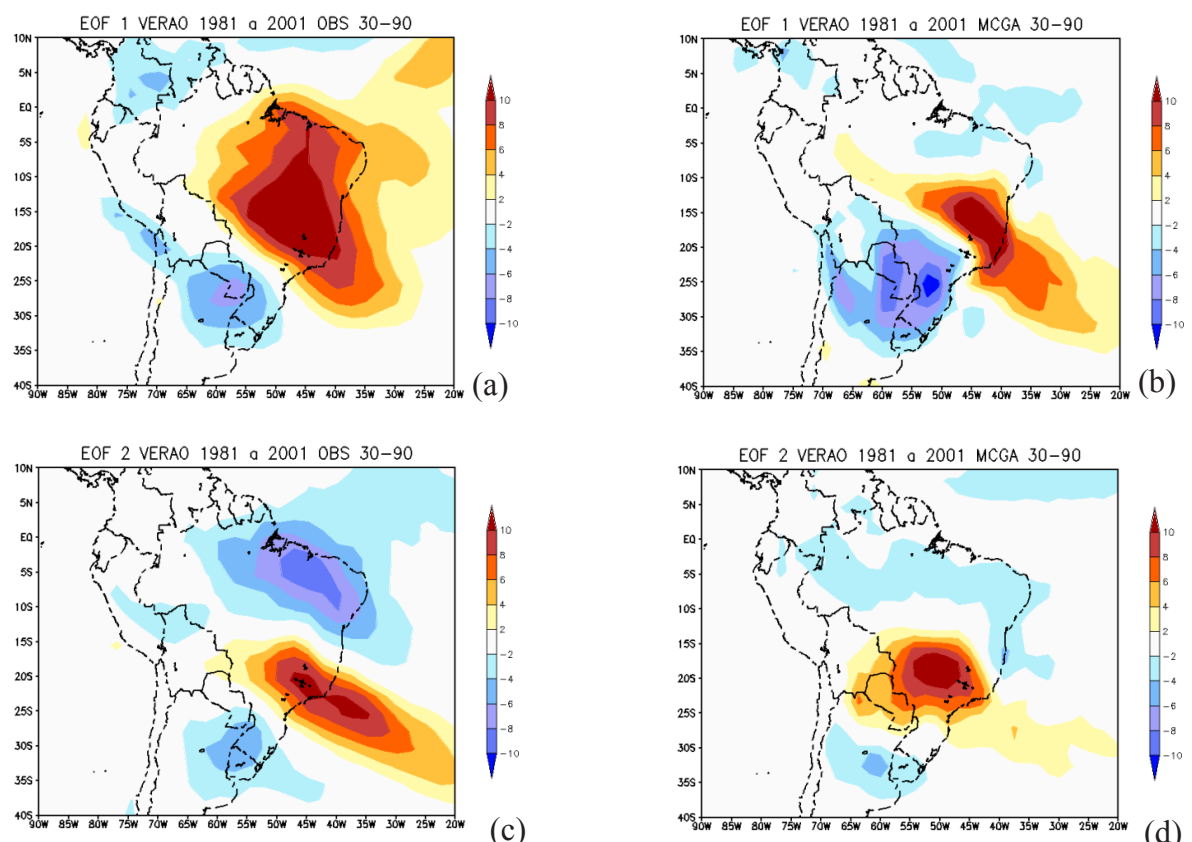

Figura 6 - EOF aplicado aos dados de anomalia de ROL na banda intrasazonal (30-90), para o verão de 1981 a 2001, na AS. (a) EOF1-OBS, (b) EOF1-MCGA, (c) EOF2-OBS e (d) EOF2-MCGA. 
As variâncias explicadas pelos dois primeiros modos de variabilidade nos resultados do modelo são bem baixas e praticamente iguais. $\mathrm{O}$ modelo simula algumas características da OMJ na região da Indonésia, e da ZCI, porém as características observadas no primeiro modo na região da ZCAS são identificadas apenas no segundo modo, com fraca intensidade (Figura 4c e d).

Análises da EOF na região tropical evidenciam o sinal da OMJ no primeiro modo de OLR observado e a sua propagação no segundo modo, porém nos resultados do modelo a configuração típica da OMJ observada não é representada, apesar de apresentar sinais alternados de anomalias sobre a Indonésia e Oceano Pacífico (Figura 5). Entretanto, quando a análise é realizada apenas para a região da $\mathrm{AS}$, o modelo simula o dipolo típico da variabilidade da ZCAS, entre o sudeste e sul, no EOF1, e o tripolo nordeste/sudeste/ sul no EOF2, porém com menor intensidade (Figura 6). Essa configuração de tripolo também foi identificada em Vasconcellos (2008) com dados de precipitação.

Muitos estudos mostram uma relação de sinais opostos de ROL sobre a AS, indicando uma tendência de gangorra na convecção (Nogués-Paegle e Mo 1997; Nogués-Paegle et al. 2000; Carvalho et al. 2004; Cunningham e Cavalcanti 2006). O modo 1 mostra dois centros com sinais opostos, um na Região Sudeste alongando-se para o Oceano Atlântico e parte do Nordeste, que indicam variações na intensidade da ZCAS, e outro sobre o Paraguai, Região Sul do Brasil, Uruguai e norte da Argentina (Figura 6a). Os resultados do modelo indicam também dois centros com sinais opostos entre a Região Sudeste e Sul, porém ampliando o centro-sul e reduzindo o centro-norte do dipolo (Figura 6b). O modelo também representa nesse primeiro modo, além da variabilidade da convecção na ZCAS, outra característica apresentada no segundo modo observado e do modelo: o sinal oposto ao do sudeste sobre o nordeste do Brasil (Figura 6c e d). Nesse segundo modo observado, a variabilidade da ZCAS, cuja convecção se estende para o oceano é bem identificada, enquanto no modelo, embora haja a configuração do tripolo observado, a convecção na ZCAS se concentra com maior intensidade sobre o continente. As características observadas são consistentes com as análises observacionais de Carvalho et al. (2004). O primeiro modo apresenta variabilidade associada à intensidade da ZCAS (categoria intensa ou fraca em Carvalho et al. 2004), enquanto o segundo modo apresenta variabilidade associada à posição (continental ou oceânica em Carvalho et al. 2004). O modelo reproduz a característica associada à intensidade da ZCAS, no EOF1, e a variabilidade da convecção sobre o continente no EOF2.

\subsection{Compostos de casos anômalos de convecção sobre a América do Sul}

Para a realização dos compostos foram selecionados os dias em que a média das anomalias filtradas em cada área de estudo (a1, a2, a3, a4, a5 e a6) encontrava-se negativa e superior ao desvio padrão de cada área (valores apresentados na Tabela I), para a observação e resultados do modelo. A Tabela I também mostra a quantidade de casos para cada área de estudo. A intenção dessa análise é verificar se há relação entre a convecção de uma área com convecção em outras áreas, como no caso do $\mathrm{sul} /$ sudeste/nordeste, e comparar os resultados do modelo com essas relações observadas.

Analisando os compostos de casos observados com convecção anômala em diferentes áreas sobre a AS, observa-se que para a área a1, que representa a Região Norte do Brasil, as maiores anomalias ocorrem no setor leste da Amazônia, se estendendo para parte do Nordeste e norte do Sudeste (Figura 7a). Entretanto, no composto com os resultados do modelo, a convecção se concentra mais sobre a área analisada, também se estendendo para o norte do Sudeste. O modelo indica uma oposição de fase sobre o centro-oeste e parte do Sudeste, realçando o fraco sinal no composto observado (Figura $7 b$ ). No composto para a área a2, além do núcleo máximo de convecção, há sinais da atuação conjunta das anomalias na ZCAS oceânica no composto observado (Figura 7a). Entretanto, no modelo, as anomalias na região da ZCAS são de sinal oposto ao da área na Região Centro-Oeste representada pela área 2 (Figura 7b). A área a3 (que engloba partes da Região Sul do Brasil, Paraguai, Uruguai e Argentina), área a4 (representando o Sudeste do Brasil) e a5 (que representa a Região Sul), têm comportamentos semelhantes, tanto nos dados observados quanto nos resultados do modelo, apresentando o dipolo de convecção associado à ZCAS, identificado em vários estudos, como mencionado na seção 3.3 (Figura 7 g-j ). A configuração dos compostos para a área 6, a qual representa a Região Nordeste, mostra uma oposição de anomalias sobre o Noroeste da Amazônia nos dados observados, enquanto no modelo essa oposição é vista sobre parte da Região Sudeste (Figura 71 e m). Assim, embora a Região Sudeste seja a região com baixa previsibilidade na escala sazonal (Marengo et al. 2003), a VI nessa região que afeta a ZCAS, é bem representada pelo MCGA.

\section{CONCLUSÕES}

Neste estudo foram apresentadas algumas características da VI de ROL simuladas pelo MCGA CPTEC/COLA e comparação com a variabilidade observada. $\mathrm{O}$ modelo reproduz as regiões com grande convecção sobre o Hemisfério Sul e região tropical, tais como a região da Indonésia, AS e África. As áreas de maior variância de ROL são também identificadas na simulação, embora com menor intensidade. Os padrões de variabilidade (EOF) são bem simulados sobre a AS (padrão de dipolo e da ZCAS), quando se considera apenas a região desse continente. Quando a área considerada abrange todo o 

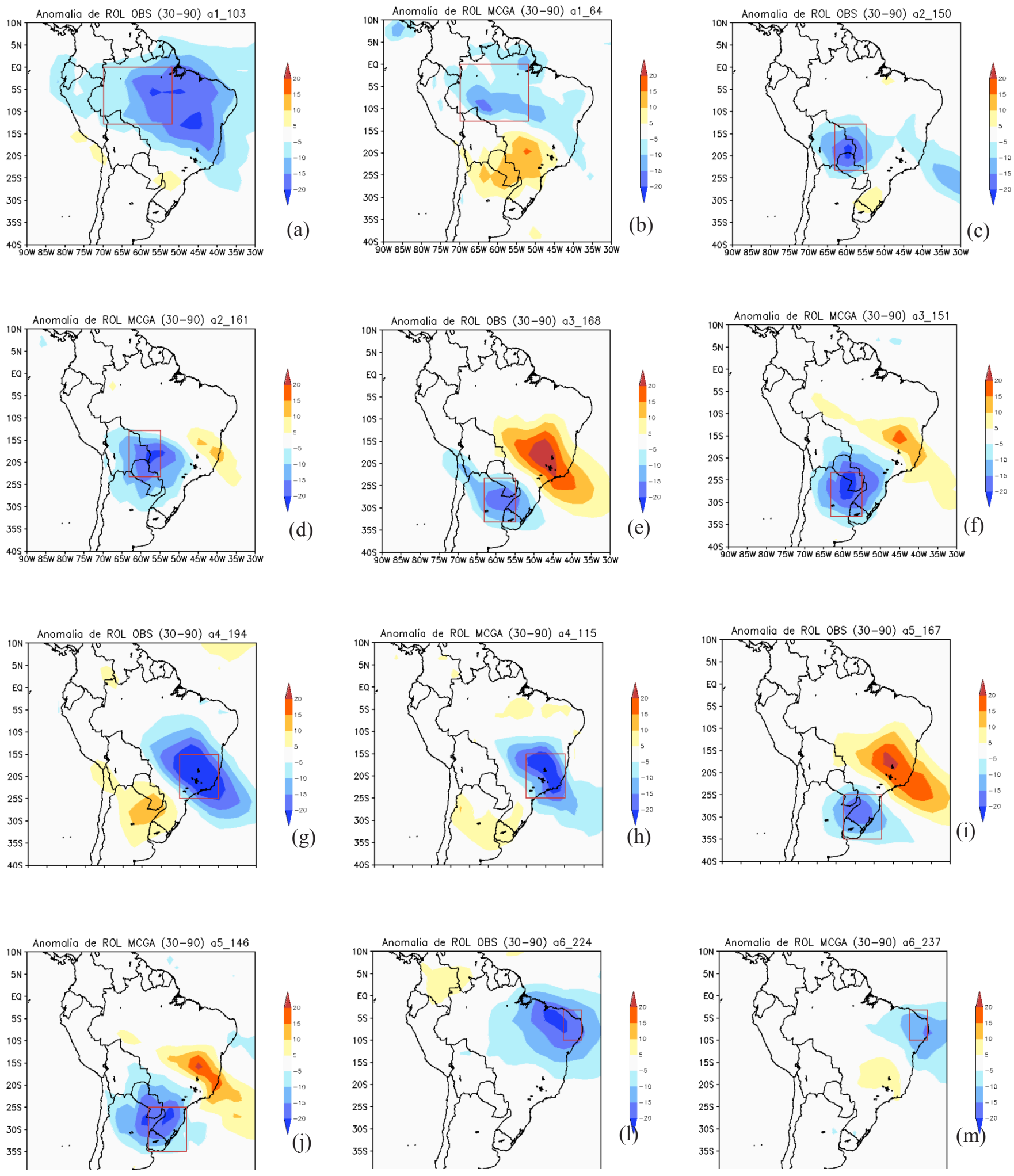

Figura 7 - Compostos para os verões de 1981 a 2001 para todas as áreas em estudo, com dados de anomalia de ROL filtrados na banda intrasazonal (30-90). OBS (a, c, e, g, i, l) MCGA (b, d, f, h, j, m). 
Hemisfério Sul ou apenas a região tropical, as configurações observadas associadas com a OMJ na região Índico/Indonésia/ Pacífico Oeste não são bem representadas. Com relação às freqüências de casos anômalos de convecção em áreas específicas, o modelo subestimou o número de casos, e as configurações dos compostos de casos com forte convecção em cada área foram melhor reproduzidas nas regiões afetadas pelo dipolo associado à ZCAS. Como a região Sudeste, afetada pela ZCAS, apresenta baixa previsibilidade mas é bem representada na VI de ROL, sugere-se que análises de VI sejam aplicadas em resultados de previsão sazonal sobre a AS, em especial sobre o Brasil.

\section{AGRADECIMENTOS}

Agradecimentos ao projeto PROTIM/CNPq, ao CNPq pelo auxílio à pesquisa, e aos revisores pelas sugestões.

\section{REFERÊNCIAS BIBLIOGRÁFICAS}

CARVALHO, L. M. V.; JONES, C.; LIEBMANN, B.: The South Atlantic Convergence Zone: Intensity, form, persistence, and relationships with intraseasonal to interannual activity and extreme rainfall. Journal of Climate. v. 17, p. 88-108, 2004.

CASARIN, D. P.; KOUSKY, V. E.: Anomalias de precipitação no sul do Brasil e variações na circulação atmosférica. Revista Brasileira de Meteorologia. v. 1, p. 83-90, 1986.

CAVALCANTI, I. F. A., CASTRO, C. C. Influências da variabilidade intrasazonal sobre a América do Sul em resultados de uma simulação climática com o MCGA CPTEC/COLA. XIII Congresso Brasileiro de Meteorologia, Fortaleza, Out. 2004.

CAVALCANTI, I. F. A.; MARENGO, J. A.; SATYAMURTY, P.; NOBRE, C. A; I. TROSNIKOV, I.; BONATTI, J. P.; MANZI, A. O.; TARASOVA, T.; PEZZI, L. P.; D'ALMEIDA, C.; SAMPAIO, G.; CASTRO, C. C.; SANCHES, M. B.; CAMARGO, H.: Global Climatological Features in a Simulation Using the CPTEC-COLA AGCM. Journal of Climate. v. 15, p. 2965-2988, 2002.

CHAVES, R. R.; NOBRE, P.: Interactions between the South Atlantic Ocean and the atmospheric circulation over South America. Geophysical Research Letters, 31, doi: 10.1029 /2003 GL018647, 2004.

CUNNINGHAM, C. C.; CAVALCANTI, I. F. A.: Intraseasonal modes of variability affecting the South Atlantic Convergence Zone. International Journal of Climatology. v. 26, p. 11651180, 2006.

DUCHON, C. E.: Lanczos filtering in one and two dimensions. Journal of Applied Meteorology, v. 18, p. 1016-1022, 1979.
HANNACHI, A., JOLLIFFE, I. T.; STEPHENSON, D. B.. Empirical orthogonal functions and related techniques in atmospheric science: A review. International Journal of Climatology,v.27,9, p.1119-1152, 2007.

KAYANO, M. T.; KOUSKY, V. E.: Sobre o monitoramento das oscilações intrasazonais. Revista Brasileira de Meteorologia. v. 7, p. 593-602, 1992.

KOUSKY, V. E.: Pentad outgoing longwave radiation climatology for the South American sector. Revista Brasileira de Meteorologia. v. 3, p. 217-231, 1988.

KOUSKY,V.E.; CAVALCANTI, I.F.A. Precipitation and atmospheric circulation anomaly patterns in the South American sector. Revista Brasileira de Meteorologia, 3, 199-206, 1988.

KOUSKY, V. E.; KAYANO, M. T.: Real-time monitoring of intraseasonal oscillations. Proc. Eighteenth Annual Climate Diagnostics Workshop, 1-5 November 1993, Boulder-CO. KOUSKY, V. E.; KAYANO, M. T.: Principal modes of outgoing longwave radiation and 250-mb circulation for the South American sector. Journal of Climate. v. 7, p. 1131-1143, 1994.

LIEBMANN, B.; SMITH C. A.: Description of a complete (Interpolated) outgoing longwave radiation dataset. Bulletin of American Meteorological Society. v. 77, p. 1275-1277, 1996.

LIEBMANN,B.;KILADIS, G.N.;MARENGO,J.A.;AMBRIZZI, T.; GLICK, J. D.: Submonthly convective variability over South America and the South Atlantic Convergence Zone. Journal of Climate. v. 12, p. 1877-1891, 1999. LIEBMANN, B.; KILADIS, G. N.; VERA, C. S.; SAULO, A. C.; CARVALHO, L. M. V.: Subseasonal variations of rainfall in South America in the vicinity of the Low-Level Jet east of the Andes and comparison to those in the South Atlantic Convergence Zone. Journal of Climate, 17, 3829-3842, 2004.

MADDEN, A. R.; JULIAN, P. R.: Observations of the 40-50-day tropical oscillation - A review. Monthly Weather Review. v. 22, p. 814-837, 1994.

MARENGO, J. A.; CAVALCANTI, I. F. A..; SATYAMURTY, P.; NOBRE, C. A.; BONATTI, J. P.; MANZI, A. O.; TROSNIKOV, I.; SAMPAIO, G.; CAMARGO, H.; SANCHES, M. B.; CUNNINGHAM, C.A. C.; D'ALMEIDA, C.; PEZZI, L. P.: Ensemble simulation of regional rainfall features in the CPTEC/COLA atmospheric GCM. Skill and predictability assessment and applications to climate predictions. Climate Dynamics, 21, 459-475, 2003.

MUZA, M. N., CARVALHO, L. M. V.; JONES, C.; LIEBMANN, B.: Intraseasonal and Interannual Variability of Extreme Dry and Wet Events over Southeastern South America and the Subtropical Atlantic during Austral Summer. Journal of Climate, 22, 1682-1699, 2009. 
NOGUÉS-PAEGLE, J.; MO, K. C.: Alternating wet and dry conditions over South America during summer. Monthly Weather Review. v. 125, p. 279-291, 1997.

NOGUÉS-PAEGLE, J. N.; BYERLE, L. A; MO, K. C.: Intraseasonal modulation of South American summer precipitation. Monthly Weather Review, v. 128, p. 837850, 2000.

SOUZA, E. B.; AMBRIZZI, T.: Modulation of the intraseasonal rainfall over tropical Brazil by the Madden-Julian oscillation. International Journal of Climatology DOI: 10.1002/joc.1331, 2006.

VASCONCELLOS, F. C.: Variabilidade atmosférica associada a casos extremos de precipitação na Região Sudeste do Brasil. 141pp. Dissertação (Mestrado em Meteorologia) - Instituto Nacional de Pesquisas Espaciais, São José dos Campos, 2008. 